Sweden). For each case one control sample was included for the ANCA specificity tests. Statistical calculations were performed using SPSS software.

Results: In ANCA-screen $36.0 \%$ of the cases and $2.6 \%$ of controls tested positive ( $p<0.001$ ). $23 / 52(44.2 \%$ ) of the cases were PR3-ANCA positive (OR 56.3; $95 \% \mathrm{Cl} 7.26-436.62)$ and 8/52 (15.4\%) were MPO-ANCA positive (OR 4.18; $95 \%$ Cl 1.05-16.62). The mean (SD) predating time for PR3-ANCA positivity was 3.73 (3.49) years and for MPO-ANCA positivity 2.11 (1.46) years. Cases with positive predating PR3-ANCA were younger $(46.0 \pm 19.4$ vs $65.6 \pm 12.0$ years; $P<0.001)$ than cases with a negative predating PR3-ANCA. MPO-ANCA positive vs. MPOANCA negative pre-dating cases had more often severe disease (kidney/lung/ peripheral nervous system) (OR 15.08; 95\% Cl 1.68-135.54) at disease onset. Furthermore, predating MPO-ANCA positive vs predating PR3-ANCA positive cases had significantly more often severe manifestations at disease onset $(87.5 \%$ vs $28.6 \%$; $p<0.05$ ). Cases positive vs. negative for MPO-ANCA in predating samples were less often classified as GPA $(37.5 \%$ vs $86.4 \%$; $<<0.01)$ and more often as MPA $(62.5 \%$ vs $13.6 \%$; $p<0.05)$.

Conclusion: The production of both PR3 and MPO-ANCA starts already years before onset of symptoms of AAV. Presence of MPO-ANCA appeared closer to symptom onset and with more severe disease presentation. Differences in the disease phenotype and disease severity were evident between the two ANCA serotypes.

References:

[1] Watts et al. Ann Rheum Dis 2007;66:222-22

Acknowledgments: : Vasculitis Foundation, USA

Disclosure of Interests: Ewa Berglin: None declared, Aladdin J Mohammad Speakers bureau: lecture fees from Roche and Elli Lilly Sweden, PI (GiACTA study), Johanna Dahlqvist: None declared, Catharina Eriksson: None declared, Johanna Sjöwall: None declared, Solbritt Rantapää Dahlqvist: None declared DOI: 10.1136/annrheumdis-2020-eular.2799

\section{SAT0254 \\ PROSPECTIVE ANALYSIS OF THE PREVALENCE OF GIANT CELL ARTERITIS IN CONSECUTIVE PATIENTS WITH POLYMYALGIA RHEUMATICA}

L. C. Burg ${ }^{1}$, C. Behning ${ }^{2}$, P. Brossart ${ }^{3}$, V. S. Schäfer ${ }^{1} .{ }^{1}$ Clinic for Internal Medicine III, University Hospital Bonn, Rheumatology, Bonn, Germany; ${ }^{2}$ University Hospital Bonn, Institute for Medical Biometry, Informatics and Epidemiology, Bonn, Germany; ${ }^{3}$ Clinic for Internal Medicine III, University Hospital Bonn, Oncology, Haematology and Rheumatoloy, Bonn, Germany

Background: Giant cell arteritis (GCA) is the most common form of systemic vasculitis affecting people aged 50 years and older. ${ }^{1}$ Although it is known, that GCA often coexists with polymyalgia rheumatica (PMR) ${ }^{2}$, prevalence of GCA in consecutive patients with PMR has not been investigated

Objectives: To prospectively examine the prevalence of GCA in consecutive patients with PMR by vascular ultrasound (US).

Methods: Patients with newly diagnosed PMR fulfilling the ACR /EULAR classification criteria $^{3}$ were included. Vascular US examination of the extracranial arteries typically involved in GCA, such as axillary arteries, vertebral arteries, common carotid arteries, superficial temporal arteries with both frontal and parietal branches, occipital arteries, facial arteries and the central retinal arteries was performed in all PMR patients. Diagnosis of GCA was made, if intima-media thickness (IMT) was above respective cut-off values. ${ }^{4}$

Results: Fifty patients with diagnosis of PMR underwent vascular US. Twenty-three patients $(46 \%)$ had PMR without GCA (PMR-group). The mean age in this group was 71 years $(S D \pm 10)$ with seventeen $(73 \%)$ females. In twenty-seven PMR patients (54\%) GCA was diagnosed (GCA-PMR group); the mean age in this group was 74 years $(S D \pm 9)$ with ten $(37 \%)$ females respectively. Mean C-reactive protein (CRP) values were $29.4 \mathrm{mg} / \mathrm{l}(\mathrm{SD} \pm 24.5)$ in the PMRgroup and $52.2 \mathrm{mg} / \mathrm{l}(\mathrm{SD} \pm 43.2)$ in the GCA-PMR-group. Although different mean values between the PMR-group and the GCA-PMR-group were observed, CRP values did not differ significantly between the two groups $(p=0.1432)$. Ten $(37 \%)$ patients of the GCA-PMR group did not have GCA symptoms and diagnosis of GCA was only determined by ultrasound examination. Symptoms and numbers of patients with respective symptoms are depicted in Table 1 and 2 .

Table 1. Symptoms and signs in both groups

\begin{tabular}{lcc}
\hline Symptoms and signs & Group & \\
\hline & PMR-group & GCA-PMR-group \\
\hline Morning stiffness & $22(95 \%)$ & $23(85 \%)$ \\
$\geq 1$ shoulder with synovits or bursitis trochanterica & $12(52 \%)$ & $13(48 \%)$ \\
$\geq 1$ shoulder or hip with synovitis or bursitis & $11(48 \%)$ & $14(51 \%)$ \\
hip pain & $23(100 \%)$ & $23(85 \%)$ \\
No other joints affected & $22(95 \%)$ & $26(96 \%)$ \\
\hline
\end{tabular}

PMR-group: patients with diagnosis of polymyalgia rheumatica only

GCA-PMR-group: patients with diagnosis of polymyalgia rheumatic and giant cell arteritis
Conclusion: Prevalence of GCA in patients with PMR in our cohort was $54 \%$. Ten (37\%) patients with GCA and PMR did not have any GCA symptoms. Performing vascular US in patients with PMR can be useful to diagnose a clinical inapparent GCA. Prompt onset of the respective therapy could prevent complications of GCA and improve disease outcome.

References:

[1] Warrington KJ, Matteson EL. Management guidelines and outcome meas ures in giant cell arteritis (GCA). Clin Exp Rheumatol 2007;25:137-41

[2] Salvarani C, Cantini F, Hunder GG. Polymyalgia rheumatica and giant-cell arteritis. The Lancet 2008;372:234-45.

[3] Dasgupta B, Cimmino MA, Kremers HM, et al. 2012 Provisional classification criteria for polymyalgia rheumatica: a European League Against Rheumatism/American College of Rheumatology collaborative initiative. Arthritis Rheum 2012;64:943-54.

[4] Schäfer VS, Juche A, Ramiro S, Krause A, Schmidt WA. Ultrasound cut-off values for intima-media thickness of temporal, facial and axillary arteries in giant cell arteritis. Rheumatology (Oxford) 2017;56:1479-83.

Table 2. Number of patients in each group with symptoms of giant cell arteritis

\begin{tabular}{lcc}
\hline Symptoms & Group & \\
\hline & PMR-group & GCA-PMR-group \\
\hline Visual symptoms & $2(9 \%)$ & $8(30 \%)$ \\
Headache & $2(9 \%)$ & $9(33 \%)$ \\
Jaw claudication & $4(18 \%)$ & $10(38 \%)$ \\
Scalp tenderness & $0(0 \%)$ & $5(19 \%)$ \\
No GCA symptoms & $15(65 \%)$ & $10(37 \%)$ \\
\hline
\end{tabular}

PMR-group: patients with diagnosis of polymyalgia rheumatica only

GCA-PMR-group: patients with diagnosis of polymyalgia rheumatic and giant cell arteritis

Disclosure of Interests: None declared

DOI: 10.1136/annrheumdis-2020-eular.5497

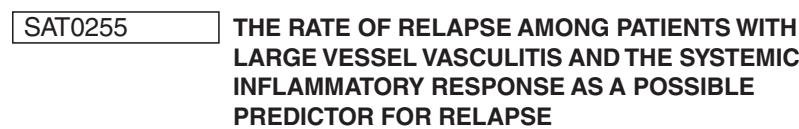

V. M. Coroian ${ }^{1}$, S. Saur ${ }^{1}$, A. C. Pecher ${ }^{1}$, T. Xenitidis ${ }^{1}$, J. Henes ${ }^{1} .{ }^{1}$ Centre for Interdisciplinary Clinical Immunology, Rheumatology and Auto-inflammatory Diseases (INDIRA) and Department of Internal Medicine II (Oncology, Haematology, Immunology and Rheumatology), University Hospital Tuebingen, Germany, Tübingen, Germany

Background: Large vessel vasculitides are known relapsing diseases. However the rate of relapses has been seldom addressed and there are only few data on relapse predictors.

Objectives: We conducted the present study to investigate the prevalence of relapses in the first year after diagnosis and the overall relapse among patients with large vessel vasculitis. Furthermore, we aimed to identify if the systemic inflammatory response (SIR) is a possible predictor for relapse among patients with large cell vasculitis

Methods: The systemic inflammatory response (SIR) has been described as a potential clinical and serological score predicting the risk for relapses ${ }^{1}$. SIR estimates the systemic inflammatory activity at the time point of first diagno$\mathrm{sis}^{1}$. It was defined as follows: Temperature $>38^{\circ} \mathrm{C}$, weight loss $>4 \mathrm{~kg}$, Haemoglobin $<11 \mathrm{~g} / \mathrm{dl}$ and erythrocyte sedimentation rate $>85 \mathrm{~mm} / \mathrm{h}$. For each of the above-mentioned criteria one point was attributed, leading to a range from 0 to 4 points. Patients with 3 to 4 points were considered having a highly inflammatory response and patients with an SIR $\leq 2$ were considered having a low inflammatory response and thus a lower risk for relapses. Relapses are defined as reappearance of disease-related symptoms requiring treatment adjustment. The study cohort included 75 patients with large cell vasculitis (Giant Cell Vasculitis Takayasu Vasculitis, inflammatory non-infections Aortitis), longitudinally followed by the authors over a mean period of $5.2 \pm 3.3$ years (range 1-14 yr).

Results: The study-cohort includes 71 patients with a mean age at diagnosis of $63.5(16-85)$ years. Almost three quarters $(73 \%)$ of the patients were women. Most of the patients were suffering from GCA (73.2\%), followed by Takayasu arteritis (16.9\%) and inflammatory non-infections Aortitis (9.8\%). 38 patients $(53.5 \%)$ relapsed at least once during the follow up, and 17 patients had two or more relapses. The vast majority of relapses $(86.8 \%)$ were observed within the first year following diagnosis. Most of the patients, 54 patients $(76 \%)$, were considered having a low inflammatory response (SIR $<=2)$. The relapse rate in this group was $59.2 \%$. On the other hand, there were 17 patients having an SIR higher or equal to 3 points. The relapse rate in this group was $33 \%$.

Conclusion: In conclusion, the results of this preliminary study reveal that the relapse rate among patients with large vessel vasculitis high is. The SRI appears to be an inadequate predictor for relapse in this cohort. 